Bull. Korean Math. Soc. 46 (2009), No. 4, pp. 645-656

DOI 10.4134/BKMS.2009.46.4.645

\title{
ON THE HYERS-ULAM-RASSIAS STABILITY OF JENSEN'S EQUATION
}

\author{
DONGYAN ZHANG AND JiAN WANG
}

\begin{abstract}
J. Wang [21] proposed a problem: whether the Hyers-UlamRassias stability of Jensen's equation for the case $p, q, r, s \in\left(\beta, \frac{1}{\beta}\right) \backslash\{1\}$ holds or not under the assumption that $G$ and $E$ are $\beta$-homogeneous $F$ space $(0<\beta \leq 1)$. The main purpose of this paper is to give an answer to Wang's problem. Furthermore, we proved that the stability property of Jensen's equation is not true as long as $p$ or $q$ is equal to $\beta, \frac{1}{\beta}$, or $\frac{\beta_{2}}{\beta_{1}}\left(0<\beta_{1}, \beta_{2} \leq 1\right)$.
\end{abstract}

\section{Introduction}

Let $G$ denote a linear space and $E$ denote a real or complex Hausdorff topological vector space. $f: \mathbf{G} \rightarrow \mathbf{E}$ is a mapping. We call the following equation

$$
2 f\left(\frac{x+y}{2}\right)-f(x)-f(y)=\theta,
$$

as Jensen's equation.

More than half a century ago, S. M. Ulam [20] posed the following problem:

Give a group $G$, and a metric group $E$ with the metric $d(\cdot, \cdot)$ and a positive number $\varepsilon>0$, does there exists a $\delta>0$ such that if a function $f: G \rightarrow E$ satisfies $d(f(x y), f(x) f(y))<\delta$ for all $x, y \in G$, then there exists a homomorphism $h: G \rightarrow E$ with $d(h(x), f(x))<\varepsilon$ for all $x \in G$ ?

In 1941, the case of approximately additive mapping was solved by D. H. Hyers [6] for $G$ and $E$ being Banach spaces. Next, Th. M. Rassias [13] generalized the conclusion of Hyers' by introducing the unbounded Cauchy difference as follows:

Received July 18, 2008

2000 Mathematics Subject Classification. 39B72.

Key words and phrases. Hyers-Ulam-Rassias stability, Jensen's functional equation.

The project supported by FPNSFC (Z0511023), the National Science Foundation of China (No.10701063) and the Foundation of Fujian Educational Committee(JA04172). 
Theorem. Let $f: G \rightarrow E$ be a mapping between Banach spaces subject to the inequality

$$
\|f(x+y)-f(x)-f(y)\| \leq \varepsilon\left(\|x\|^{p}+\|y\|^{p}\right) \quad(\forall x, y \in G),
$$

where $\varepsilon, p$ are constants with $\varepsilon>0$ and $0 \leq p<1$. Then there exists a unique additive mapping $T: G \rightarrow E$ such that

$$
\|f(x)-T(x)\| \leq \frac{2 \varepsilon}{2-2^{p}}\|x\|^{p} \quad(\forall x \in G) .
$$

If, in addition $f(t x)$ is continuous in $t$ for each fixed $x \in G$, then $T$ is linear.

The proof given in [13] also works when $p<0$. In 1991, Z. Gajda [3] following the spirit of the proof of Th. M. Rassias's Theorem for the unbounded Cauchy difference by replacing $n$ by $-n$ solved Th. M. Rassias's question by proving the stability theorem for all real values of $p$ that are strictly greater than one. And in this paper, Z. Gajda found firstly that the stability problem does not hold when $p=1$.

The remarkable generalization of Th. M. Rassias for D. H. Hyer's Theorem promoted greatly the development of the stability problems of functional equations. It stimulated a number of mathematicians to study the stability problems of various functional equations. For more detailed information of such a field one can refer to [4], [14], [15], and [16].

In this paper, we deal with the stability of the Jensen's functional Eq.(1).

The first result on the stability of Jensen's equation was carried out by Z. Kominek [9]. New generalizations of Jensen's functional equation were given by Th. M. Rassias [12]. In 1998, S.-M. Jung [8] gave an important generalization of the Z. Kominek's result. In fact, he proved the following theorem:

Theorem. Let $E_{1}$ be a real normed space and let $E_{2}$ be a real Banach space. Assume that $\delta, \theta \geq 0$ are fixed, and let $p>0$ be given with $p \neq 1$. Suppose a mapping $f: E_{1} \rightarrow E_{2}$ satisfied the functional inequality

$$
\left\|2 f\left(\frac{x+y}{2}\right)-f(x)-f(y)\right\| \leq \delta+\theta\left(\|x\|^{p}+\|y\|^{p}\right)
$$

for all $x, y \in E_{1}$. Furthermore, assume $f(0)=0$ and $\delta=0$ in above inequality for the case of $p>1$. Then there exists a unique additive function $T: E_{1} \rightarrow E_{2}$ such that

$$
\|f(x)-T(x)\| \leq \delta+\|f(0)\|+\frac{\theta}{2^{1-p}-1}\|x\|^{p} \quad(\text { for } p<1)
$$

or

$$
\|f(x)-T(x)\| \leq \frac{2^{p-1} \theta}{2^{p-1}-1}\|x\|^{p} \quad(\text { for } p>1)
$$

for all $x \in E_{1}$.

Later, many results concerning the stability of Jensen's equation were obtained by numerous authors, such as [11], [19], and [10]. J. Wang [22], [24] 
attempted to weaken the condition of the space. She proved a generalized conclusion of S.-M. Jung. In the following, we introduce Wang's result [24]:

Corollary I. Let $G$ be an $F^{*}$-space and $E$ be an $F$-space with the property that there exists $0<\beta \leq 1$ such that $\left\|\frac{u}{3}\right\| \leq \frac{\|u\|}{3^{\beta}}$ for all $u \in E$. If $\phi(x, y)=$ $\delta+\varepsilon_{1}\|x\|^{p}+\varepsilon_{2}\|y\|^{q}\left(\delta, \varepsilon_{1}, \varepsilon_{2} \geq 0, p, q<\beta\right)$, then there exists a unique additive mapping $T: G \rightarrow E$ such that

$$
\begin{aligned}
& \|T(x)-f(x)+f(\theta)\| \\
\leq & \frac{2 \delta}{3^{\beta}-1}+\frac{2 \varepsilon_{1}}{3^{\beta}-3^{p}}\|x\|^{p}+\frac{\left(1+3^{q}\right) \varepsilon_{2}}{3^{\beta}-3^{q}}\|x\|^{q}
\end{aligned}
$$

for any $x \in G$. If there exists at least one of $p, q$ such that it is strictly less than 0 , then the domain of $T$ is $G \backslash\{\theta\}$ instead of $G$.

Corollary II. Let $G$ be an $F^{*}$-space with the property that there exists $0<$ $\beta \leq 1$ such that $\left\|\frac{x}{3}\right\| \leq \frac{\|x\|}{3^{\beta}}$ for all $x \in G$, and $E$ be an $F$-space. Assume that $f(\theta)=\theta$. If $\phi(x, y)=\delta+\varepsilon_{1}\|x\|^{p}+\varepsilon_{2}\|y\|^{q}\left(\varepsilon_{1}, \varepsilon_{2} \geq 0, p, q>\frac{1}{\beta}\right)$, then there exists a unique additive mapping $T: G \rightarrow E$ such that

$$
\begin{aligned}
& \|T(x)-f(x)\| \\
\leq & \frac{2 \varepsilon_{1}}{3^{p \beta}-3}\|x\|^{p}+\frac{\left(1+3^{q \beta}\right) \varepsilon_{2}}{3^{q \beta}-3}\|x\|^{q}
\end{aligned}
$$

for any $x \in G$.

In above corollaries, $\phi(x, y)=\frac{1}{2} f\left(\frac{x+y}{2}\right)-f(x)-f(y)$.

J. Wang noticed that these results hold for $p, q<\beta$ or $p, q>\frac{1}{\beta}$. She raised the following question: What does it hold if $p, q$ satisfy $\beta<p, q<\frac{1}{\beta}$ under the assumption that $G$ and $E$ are $\beta$-homogeneous $F$-spaces $(0<\beta \leq 1)$ ? In Section 2 of the present paper, by still using the ideas from the papers of Hyers [6], Rassias [13], Rassias and S̆emrl [16], we provide the stability of Eq.(1) for $\beta_{2}<p, q<\frac{1}{\beta_{1}}\left(p, q \neq \frac{\beta_{2}}{\beta_{1}}\right)$ in $\beta$-homogeneous $F$-space. In Section 3, we show that the stability of Jensen's equation is not satisfied as long as $p$ or $q$ equals $\beta_{2}, \frac{1}{\beta_{1}}$ or $\frac{\beta_{2}}{\beta_{1}}\left(0<\beta_{1}, \beta_{2} \leq 1\right)$.

$$
\text { 2. Stability of Eq.(1) for } \beta_{2}<p, q<\frac{1}{\beta_{1}}\left(p, q \neq \frac{\beta_{2}}{\beta_{1}}\right)
$$

From now on, we let $\mathbb{N}$ denote the set of positive integers set and $\mathbb{R}$ denotes the set of real numbers set, respectively. Meanwhile, We assume $p, q$ to be different real numbers.

Firstly, we introduce the definition of $F$-space and $\beta$-homogeneous (see [18]).

Let $X$ be a linear space. A non-negative valued function $\|\cdot\|$ defined on $X$ is called an $F$-norm if it obeys the following rules:
(n1) $\quad\|x\|=0$ if and only if $x=0$;
(n2) $\quad\|a x\|=\|x\|$ for all $a,|a|=1$;
(n3) $\|x+y\| \leq\|x\|+\|y\|$; 
(n4) $\quad\left\|a_{n} x\right\| \longrightarrow 0$ provided $a_{n} \longrightarrow 0$;

(n5) $\left\|a x_{n}\right\| \longrightarrow 0$ provided $x_{n} \longrightarrow 0$.

A space $X$ with an $F$-norm is called an $F^{*}$-space. An $F$-pseudonorm $(\|x\|=$ 0 does not necessarily imply that $x=0$ in $(\mathrm{n} 1))$ is called $\beta$-homogeneous $(\beta>0)$ if $\|t x\|=|t|^{\beta}\|x\|$ for all $x \in X$ and all $t \in \mathbb{R}$. A complete $F^{*}$-space is said to be an $F$-space.

Theorem 2.1. Let $G$ and $E$ be a $\beta_{1}$-homogeneous $F^{*}$-space and a $\beta_{2}$-homogeneous $F$-space, respectively. Suppose that $f: G \rightarrow E$ is a mapping with the property that

$$
\left\|2 f\left(\frac{x+y}{2}\right)-f(x)-f(y)\right\| \leq \varepsilon_{1}\|x\|^{p}+\varepsilon_{2}\|y\|^{q},
$$

where $\beta_{1}, \beta_{2} \in(0,1], \varepsilon_{1}, \varepsilon_{2} \in(0, \infty)$ and $p, q \in\left(\beta_{2}, \frac{1}{\beta_{1}}\right) \backslash\left\{\frac{\beta_{2}}{\beta_{1}}\right\}$. Then there exists a unique additive mapping $T: G \rightarrow E$ such that

$$
\|T(x)-f(x)\| \leq \frac{2 \varepsilon_{1}}{3^{\beta_{2}}-3^{\beta_{1} p}}\|x\|^{p}+\frac{\left(1+3^{\beta_{1} q}\right) \varepsilon_{2}}{3^{\beta_{2}}-3^{\beta_{1} q}}\|x\|^{q}
$$

in the case $\beta_{2}<p, q<\frac{\beta_{2}}{\beta_{1}}$, while in the case $\frac{\beta_{2}}{\beta_{1}}<p, q<\frac{1}{\beta_{1}}$

$$
\|T(x)-f(x)\| \leq \frac{2 \varepsilon_{1}}{3^{\beta_{1} p}-3^{\beta_{2}}}\|x\|^{p}+\frac{\left(1+3^{\beta_{1} q}\right) \varepsilon_{2}}{3^{\beta_{1} q}-3^{\beta_{2}}}\|x\|^{q} .
$$

Moreover, if for each fixed $x \in G$, there exists a real numbers $\delta_{x}>0$, such that $f(t x)$ is continuous on $\left[0, \delta_{x}\right]$, then $T(x)$ is linear.

Proof. Let $g(x)=f(x)-f(\theta)$. Then $g$ also satisfies (2). From this, we can assume that $f(\theta)=\theta$ without loss of generality.

When $\beta_{2}<p, q<\frac{\beta_{2}}{\beta_{1}}$, we claim that

$$
\begin{aligned}
& \left\|3^{-n} f\left(3^{n}\right)-f(x)\right\| \\
\leq & \sum_{k=1}^{n} 3^{k\left(\beta_{1} p-\beta_{2}\right)} \cdot 2 \cdot 3^{-\beta_{1} p} \varepsilon_{1}\|x\|^{p} \\
& +\sum_{k=1}^{n}\left(3^{k\left(\beta_{1} q-\beta_{2}\right)} \cdot 3^{-\beta_{1} q}+3^{k\left(\beta_{1} q-\beta_{2}\right)}\right) \varepsilon_{2}\|x\|^{q}
\end{aligned}
$$

holds for any integer $n$. The verification of (5) follows by induction on $n$. Indeed, for $n=1$, we set $y=-x$, then

$$
\|-f(x)-f(-x)\| \leq \varepsilon_{1}\|x\|^{p}+\varepsilon_{2}\|x\|^{q} .
$$

Replacing $x$ by $-x$ and $y$ by $3 x$, (5) implies

$$
\|2 f(x)-f(-x)-f(3 x)\| \leq \varepsilon_{1}\|x\|^{p}+\varepsilon_{2} \cdot 3^{\beta_{1} q}\|x\|^{q} .
$$


Taking the two inequality into account, then

$$
\begin{aligned}
\left\|3^{-1} f(3 x)-f(x)\right\| & =\left\|3^{-1}[f(3 x)+f(-x)-2 f(x)-f(-x)-f(x)]\right\| \\
& \leq 3^{-\beta_{2}}[\|f(3 x)+f(-x)-2 f(x)\|+\|-f(-x)-f(x)\|] \\
& \leq 2 \cdot 3^{-\beta_{2}} \cdot \varepsilon_{1}\|x\|^{p}+\left(1+3^{\beta_{1} q}\right) \cdot 3^{-\beta_{2}} \varepsilon_{2}\|x\|^{q} .
\end{aligned}
$$

Assume that the formula is true for $n=m$, we want to examine the case when $n=m+1$. We have

$$
\begin{aligned}
& \left\|3^{-(m+1)} f\left(3^{(m+1)} x\right)-f(x)\right\| \\
= & \left\|3^{-1}\left[3^{-m} f\left(3^{m}(3 x)\right)-f(3 x)\right]+3^{-1} f(3 x)-f(x)\right\| \\
\leq & 3^{-\beta_{2}}\left[\sum_{k=1}^{m} 3^{k\left(\beta_{1} p-\beta_{2}\right)} \cdot 2 \cdot 3^{-\beta_{1} p} \varepsilon_{1}\|3 x\|^{p}+\sum_{k=1}^{m}\left(3^{k\left(\beta_{1} q-\beta_{2}\right)} \cdot 3^{-\beta_{1} q}+3^{k\left(\beta_{1} q-\beta_{2}\right)}\right) \varepsilon_{2}\|3 x\|^{q}\right] \\
& +2 \cdot 3^{-\beta_{2}} \cdot \varepsilon_{1}\|x\|^{p}+\left(1+3^{\beta_{1} q}\right) \cdot 3^{-\beta_{2}} \varepsilon_{2}\|x\|^{q} \\
= & \sum_{k=1}^{m+1} 3^{k\left(\beta_{1} p-\beta_{2}\right)} \cdot 2 \cdot 3^{-\beta_{1} p} \varepsilon_{1}\|x\|^{p}+\sum_{k=1}^{m+1}\left(3^{k\left(\beta_{1} q-\beta_{2}\right)} \cdot 3^{-\beta_{1} q}+3^{k\left(\beta_{1} q-\beta_{2}\right)}\right) \varepsilon_{2}\|x\|^{q} .
\end{aligned}
$$

Therefore (5) is proved.

$$
\text { Let }
$$

$$
T(x)=\lim _{n \rightarrow \infty} \frac{f\left(3^{n} x\right)}{3^{n}} .
$$

It is easy to see that $T$ exists. In fact,

$$
\begin{aligned}
& \left\|\frac{f\left(3^{m} x\right)}{3^{m}}-\frac{f\left(3^{n} x\right)}{3^{n}}\right\| \\
= & \left\|\frac{1}{3^{n}}\left[\frac{f\left(3^{m-n}\left(3^{n} x\right)\right)}{3^{m-n}}-f\left(3^{n} x\right)\right]\right\| \\
\leq & \frac{1}{3^{n \beta_{2}}}\left[\sum_{k=1}^{m-n} 3^{k\left(\beta_{1} p-\beta_{2}\right)} 2 \cdot 3^{-\beta_{1} p} \varepsilon_{1}\left\|3^{n} x\right\|^{p}+\sum_{k=1}^{m-n}\left(3^{k\left(\beta_{1} q-\beta_{2}\right)} \cdot 3^{-\beta_{1} q}+3^{k\left(\beta_{1} q-\beta_{2}\right)}\right) \varepsilon_{2}\left\|3^{n} x\right\|^{q}\right] \\
\leq & \frac{1}{3^{n\left(\beta_{2}-\beta_{1} p\right)}}\left[\frac{2 \varepsilon_{1}}{3^{\beta_{2}}-3^{\beta_{1} p}}\|x\|^{p}+\frac{\left(1+3^{\beta_{1} q}\right) \varepsilon_{2}}{3^{\beta_{2}}-3^{\beta_{1} q}}\|x\|^{q}\right]
\end{aligned}
$$

for any $m>n, m, n \in \mathbb{N}$. By virtue of $\beta_{2}-\beta_{1} p>0$, it follows that

$$
\lim _{n \rightarrow \infty}\left\|\frac{f\left(3^{m} x\right)}{3^{m}}-\frac{f\left(3^{n} x\right)}{3^{n}}\right\|=0 .
$$

Thus $\left\{\frac{f\left(3^{n} x\right)}{3^{n}}\right\}$ is a Cauchy sequence. However the $F$-space is complete, thus $\left\{\frac{f\left(3^{n} x\right)}{3^{n}}\right\}$ converges. It follows that $T(x)=\lim _{n \rightarrow \infty} \frac{f\left(3^{n} x\right)}{3^{n}}$ exists. Hence by letting $n \rightarrow \infty$ in (5), one obtains

$$
\|T(x)-f(x)\| \leq \frac{2 \varepsilon_{1}}{3^{\beta_{2}}-3^{\beta_{1} p}}\|x\|^{p}+\frac{\left(1+3^{\beta_{1} q}\right) \varepsilon_{2}}{3^{\beta_{2}}-3^{\beta_{1} q}}\|x\|^{q} .
$$


Now we shall deal with the additivity of $T$. On account of (6), one has

$$
T\left(3^{m} x\right)=\lim _{n \rightarrow \infty} \frac{f\left(3^{n}\left(3^{m} x\right)\right)}{3^{n+m}} \cdot 3^{m}=3^{m} T(x) .
$$

And employing the condition (2), we set

$$
\begin{aligned}
& \left\|2 T\left(\frac{x+y}{2}\right)-T(x)-T(y)\right\| \\
= & \lim _{n \rightarrow \infty}\left\|2 \cdot \frac{1}{3^{n}} f\left(\frac{3^{n} x+3^{n} y}{2}\right)-\frac{1}{3^{n}} f\left(3^{n} x\right)-\frac{1}{3^{n}} f\left(3^{n} y\right)\right\| \\
\leq & \lim _{n \rightarrow \infty}\left(\frac{\varepsilon_{1}}{3^{n\left(\beta_{2}-\beta_{1} p\right)}}\|x\|^{p}+\frac{\varepsilon_{2}}{3^{n\left(\beta_{2}-\beta_{1} q\right)}}\|y\|^{q}\right) \\
= & 0 .
\end{aligned}
$$

By (3), (6), and (7), it follows

$$
\begin{aligned}
& \|2 T(2 x)-4 T(x)\|=\|2 T(2 x)-T(3 x)-T(x)\| \\
& =\left\|3^{-n}\left[2 T\left(3^{n} \cdot 2 x\right)-T\left(3^{n} \cdot 3 x\right)-T\left(3^{n} x\right)\right]\right\| \\
& \leq 3^{-n \beta_{2}}\left(\left\|2 T\left(3^{n} \cdot 2 x\right)-2 f\left(3^{n} \cdot 2 x\right)\right\|+\left\|T\left(3^{n} \cdot 3 x\right)-f\left(3^{n} \cdot 3 x\right)\right\|\right) \\
& +3^{-n \beta_{2}}\left(\left\|T\left(3^{n} x\right)-f\left(3^{n} x\right)\right\|+\left\|2 f\left(\frac{3^{n}(3 x+x)}{2}\right)-f\left(3^{n} \cdot 3 x\right)-f\left(3^{n} x\right)\right\|\right) \\
& \leq \frac{2 \varepsilon_{1}\|x\|^{p}}{3^{n\left(\beta_{2}-\beta_{1} p\right) \cdot\left(3^{\beta_{2}}-3^{\beta_{1} p}\right)}}\left(2^{\beta_{1} p+\beta_{2}}+3^{\beta_{1} p}+1\right) \\
& +\frac{\left(1+3^{\beta_{1} q}\right) \varepsilon_{2}\|x\|^{q}}{3^{n\left(\beta_{2}-\beta_{1} q\right) \cdot\left(3^{\beta_{2}}-3^{\beta_{1} p}\right)}}\left(2^{\beta_{1} q+\beta_{2}}+3^{\beta_{1} q}+1\right) \\
& +\frac{3^{\beta_{1} p} \cdot \varepsilon_{1}}{3^{n\left(\beta_{2}-\beta_{1} p\right)}}\|x\|^{p}+\frac{\varepsilon_{2}}{3^{n\left(\beta_{2}-\beta_{1} q\right)}}\|x\|^{q} .
\end{aligned}
$$

Clearly, $\|2 T(2 x)-4 T(x)\| \rightarrow 0$ as $n \rightarrow \infty$. Thus, $2 T(2 x)=4 T(x)$. From (8), we get

$$
T(x+y)=\frac{1}{2}(T(2 x)+T(2 y))=T(x)+T(y) .
$$

We will prove the uniqueness of $T$. Suppose that $H: G \rightarrow E$ is another additive mapping satisfying (3) for all $x \in G$. It follows that

$$
\begin{aligned}
& \|T(x)-H(x)\|=\frac{1}{n^{\beta_{2}}}\|T(n x)-H(n x)\| \\
= & \frac{1}{n^{\beta_{2}}}\|T(n x)-f(n x)-H(n x)+f(n x)\| \\
\leq & \frac{1}{n^{\beta_{2}}}(\|T(n x)-f(n x)\|+\|H(n x)-f(n x)\|) \\
\leq & \frac{4 \varepsilon_{1}\|x\|^{p}}{n^{\left(\beta_{2}-\beta_{1} p\right)} \cdot\left(3^{\beta_{2}}-3^{\beta_{1} p}\right)}+\frac{2\left(1+3^{\beta_{1} q}\right) \varepsilon_{2}\|x\|^{q}}{n^{\left(\beta_{2}-\beta_{1} q\right)} \cdot\left(3^{\beta_{2}}-3^{\beta_{1} q}\right)},
\end{aligned}
$$

and so $\|T(x)-H(x)\| \rightarrow 0$ as $n \rightarrow \infty$. Hence $T(x)=H(x)$ for all $x \in G$.

This finishes the first step of the proof. 
When $\frac{\beta_{2}}{\beta_{1}}<p, q<\frac{1}{\beta_{1}}$, we claim that

$$
\begin{aligned}
& \left\|3^{n} f\left(3^{-n}\right)-f(x)\right\| \\
\leq & \sum_{k=1}^{n} 3^{k\left(\beta_{2}-\beta_{1} p\right)} \cdot 2 \cdot 3^{-\beta_{2}} \varepsilon_{1}\|x\|^{p} \\
& +\sum_{k=1}^{n}\left(3^{k\left(\beta_{2}-\beta_{1} q\right)} \cdot 3^{-\beta_{2}}+3^{(k-1)\left(\beta_{2}-\beta_{1} q\right)}\right) \varepsilon_{2}\|x\|^{q} .
\end{aligned}
$$

Note that substituting $3^{-n} x$ by $x$ in (5) and later multiplying both sides by $3^{n \beta_{2}}$, we can yield the above formula (9).

Define $T(x)=\lim _{n \rightarrow \infty} 3^{n} f\left(3^{-n} x\right)$. The rest of the proofs follows as that in the case of $\beta_{2}<p, q<\frac{\beta_{2}}{\beta_{1}}$, and therefore we omit it.

Consequently, we obtain

$$
\|T(x)-f(x)\| \leq \frac{2 \varepsilon_{1}}{3^{\beta_{1} p}-3^{\beta_{2}}}\|x\|^{p}+\frac{\left(1+3^{\beta_{1} q}\right) \varepsilon_{2}}{3^{\beta_{1} q}-3^{\beta_{2}}}\|x\|^{q} .
$$

Moreover, if for each fixed $x \in G$, there exists a real number $\delta_{x}>0$, such that $f(t x)$ is continuous on $\left[0, \delta_{x}\right]$, we claim that $f(t x)$ is bounded on $\left[0, \delta_{x}\right]$. Otherwise, if this were not the case then for any $n \in \mathbb{N}$, there exists $t_{n} \in\left[0, \delta_{x}\right]$ such that $\left\|f\left(t_{n} x\right)\right\| \geq n$. For the bounded sequence $\left\{t_{n}\right\}$, we could apply the Bolzano-Weierstass theorem to find a convergent subsequence $\left\{t_{n_{k}}\right\}$ and $t_{0} \in$ $\left[0, \delta_{x}\right]$ such that $\lim _{k \rightarrow \infty} t_{n_{k}}=t_{0}$. It follows that $\lim _{k \rightarrow \infty} t_{n_{k}} x=t_{0} x$ for each fixed $x \in G$. Since $f(t x)$ is continuous in $t_{0}$, we can conclude that $\lim _{k \rightarrow \infty} f\left(t_{n_{k}} x\right)=$ $f\left(t_{0} x\right)$. Thus, we get a contradiction to $\lim _{k \rightarrow \infty}\left\|f\left(t_{n_{k}}\right)\right\|=\infty$. The remaining proof follows a similar argument as in the proof of [16], hence we obtain that $T(x)$ is linear. Thus, claim is given.

Remark 1. Let $G$ and $E$ be a $\beta_{1}$-homogeneous $F^{*}$-space and a $\beta_{2}$-homogeneous $F$-space, respectively. Suppose that $f: G \rightarrow E$ satisfies

$$
\left\|2 f\left(\frac{x+y}{2}\right)-f(x)-f(y)\right\| \leq \delta .
$$

Then there exists a unique additive mapping $T: G \rightarrow E$ such that

$$
\|T(x)-f(x)\| \leq \frac{2 \delta}{3^{\beta_{2}}-1}
$$

for all $x \in G$.

Now we construct an $F$-norm satisfying the condition that there exists $0<$ $\beta<1$ such that $\left\|\frac{x}{3}\right\| \leq \frac{\|x\|}{3^{\beta}}$ but not $\beta$-homogeneity. So, the condition of spaces $G$ and $E$ in theorem can be weakened. 
Example 1. We define the non-negative function $\|\cdot\|$ in $\mathbb{R}$ by

$$
\|x\|=\left\{\begin{array}{ll}
|x|^{\beta} & |x| \leq 1 \\
|x| & |x|>1
\end{array} \quad(\forall x \in \mathbb{R}) .\right.
$$

Then $\|\cdot\|$ is an $F$-norm with the property that $\left\|\frac{x}{n}\right\| \leq \frac{\|x\|}{n^{\beta}}(n \in \mathbb{N})$, but not the $\beta$-homogeneity.

Proof. We have only to show that $\|\cdot\|$ satisfies the triangle inequality. To establish one, we shall consider three cases. In the case where $|x|>1,|y|>1$, one has

$$
\|x+y\|=|x+y| \leq|x|+|y|=\|x\|+\|y\| .
$$

In the case where $|x|<1,|y|<1$, and likewise $|x+y| \leq 1$,

$$
\|x+y\|=|x+y|^{\beta} \leq(|x|+|y|)^{\beta} \leq|x|^{\beta}+|y|^{\beta}=\|x\|+\|y\|,
$$

or $|x|<1,|y|<1$ and likewise $|x+y|>1$, and therefore

$$
\|x+y\|=|x+y| \leq|x|+|y| \leq|x|^{\beta}+|y|^{\beta}=\|x\|+\|y\| .
$$

While in the case where $|x|>1,|y|<1$ or $|x|<1,|y|>1$, we might as well suppose that $|x|>1,|y|<1$. Then if $|x+y| \leq 1$ holds, we obtain

$$
\|x+y\|=|x+y|^{\beta} \leq|x|^{\beta}+|y|^{\beta} \leq|x|+|y|^{\beta}=\|x\|+\|y\| .
$$

However, if $|x+y|>1$ then,

$$
\|x+y\|=|x+y| \leq|x|+|y| \leq|x|+|y|^{\beta}=\|x\|+\|y\| .
$$

Therefore $\|\cdot\|$ is an $F$-norm.

Now we will prove that $\left\|\frac{x}{n}\right\| \leq \frac{\|x\|}{n^{\beta}}$ for any $n \in \mathbb{N}$. Indeed, when $|x| \leq n$, then

$$
\left\|\frac{x}{n}\right\|=\left|\frac{x}{n}\right|^{\beta}=\frac{1}{n^{\beta}}|x|^{\beta}=\frac{1}{n^{\beta}}\|x\|^{\beta}
$$

and also when $|x|>n$, one has

$$
\left\|\frac{x}{n}\right\|=\frac{|x|}{n} \leq \frac{|x|}{n^{\beta}}=\frac{\|x\|}{n^{\beta}} .
$$

It follows that $\left\|\frac{x}{n}\right\| \leq \frac{\|x\|}{n^{\beta}}$ for any $x \in \mathbb{R}$.

It is easy to see that the $\|\cdot\|$ is not $\beta$-homogeneous.

Therefore the proof is completed.

\section{Instability of Eq.(1)}

We will first cite the counterexample constructed by Z. Gajda [3].

Example 2. For a fixed $\varepsilon>0$ and $\mu=\frac{\varepsilon}{6}$, define a function $f: \mathbb{R} \rightarrow \mathbb{R}$ by

$$
f(x)=\sum_{n=0}^{\infty} \frac{\phi\left(2^{n} x\right)}{2^{n}} \quad x \in \mathbb{R},
$$


where the function $\phi: \mathbb{R} \rightarrow \mathbb{R}$ is given by

$$
\phi(x)=\left\{\begin{array}{cc}
\mu & x \leq 1 \\
\mu x & -1<x<1 \\
-\mu & x \leq-1
\end{array}\right.
$$

Theorem 3.1. The function $f$ defined above satisfies

$$
|f(x+y)-f(x)-f(y)| \leq \varepsilon\left(|x|+|y|^{\frac{1}{2}}\right)
$$

for all $x, y \in \mathbb{R}$. However

$$
\sup \left\{\frac{|f(x)-T(x)|}{|x|}: x \in \mathbb{R} \backslash\{0\}\right\}=\infty
$$

for each additive mapping $T: \mathbb{R} \rightarrow \mathbb{R}$.

Proof. The inequality (10) is trivially fulfilled if $x=y=0$.

Now, we assume that $|x|+|y|^{\frac{1}{2}}<1$. Then $|x|<1,|y|^{\frac{1}{2}}<1$. There exists an $N \in \mathbb{N}$ such that

$$
2^{N-1}\left(|x|+|y|^{\frac{1}{2}}\right)<1, \quad 2^{N}\left(|x|+|y|^{\frac{1}{2}}\right) \geq 1 .
$$

Since $|x|+|y| \leq|x|+|y|^{\frac{1}{2}}$, we get $2^{N-1}(|x|+|y|) \leq 2^{N-1}\left(|x|+|y|^{\frac{1}{2}}\right)<1$. Hence,

$$
\left|2^{N-1}(x+y)\right| \leq 2^{N-1}(|x|+|y|)<1 \quad \text { and } \quad\left|2^{N-1} x\right|<1, \quad\left|2^{N-1} y\right|<1,
$$

which means that for each $n \in\{0,1,2, \ldots, N-1\}, 2^{n-1} x, 2^{n-1} y, 2^{n-1}(x+y) \in$ $(-1,1)$. Since $\phi$ is a linear mapping on the interval, we infer that

$$
\phi\left(2^{n}(x+y)\right)=\phi\left(2^{n} x\right)+\phi\left(2^{n} y\right)
$$

for $n=0,1, \ldots, N-1$. As a result, we obtain

$$
\begin{aligned}
\frac{|f(x+y)-f(x)-f(y)|}{|x|+|y|^{\frac{1}{2}}} & \leq \sum_{n=0}^{\infty} \frac{\left|\phi\left(2^{n}(x+y)\right)-\phi\left(2^{n} x\right)-\phi\left(2^{n} y\right)\right|}{2^{n}\left(|x|+|y|^{\frac{1}{2}}\right)} \\
& =\sum_{n=N}^{\infty} \frac{\left|\phi\left(2^{n}(x+y)\right)-\phi\left(2^{n} x\right)-\phi\left(2^{n} y\right)\right|}{2^{n}\left(|x|+|y|^{\frac{1}{2}}\right)} \\
& \leq \sum_{k=0}^{\infty} \frac{3 \mu}{2^{k} \cdot 2^{N}\left(|x|+|y|^{\frac{1}{2}}\right)} \leq \sum_{k=0}^{\infty} \frac{3 \mu}{2^{k}}=6 \mu .
\end{aligned}
$$

Finally, assume that $|x|+|y|^{\frac{1}{2}} \geq 1$. Then because of the boundedness of $f$, we have

since

$$
\frac{|f(x+y)-f(x)-f(y)|}{|x|+|y|^{\frac{1}{2}}} \leq 6 \mu=\varepsilon,
$$

$$
|f(x)| \leq \sum_{n=0}^{\infty}=2 \mu, \quad x \in \mathbb{R} .
$$

Thus, we conclude that $f$ satisfies (10) for all $x, y \in \mathbb{R}$. The proof of the last assertion in the theorem follows the same argument as in [3]. 
Remark 2. Let the function $f$ be as before.

(i) If $G=\left(\mathbb{R},\|\cdot\|_{1}\right)$ with the Euclidean metric $\|\cdot\|_{1}=|\cdot|$ and $E=\left(\mathbb{R},\|\cdot\|_{2}\right)$ with the $\beta$-homogeneous norm $\|\cdot\|_{2}=|\cdot|^{\beta}$, then

$$
\|f(x+y)-f(x)-f(y)\|_{2} \leq \varepsilon^{\beta}\left(\|x\|_{1}^{\beta}+\|y\|_{1}^{\frac{\beta}{2}}\right)
$$

for any $x, y \in \mathbb{R}$, however

$$
\sup \left\{\frac{\|f(x)-T(x)\|_{2}}{\|x\|_{1}^{\beta}}: x \in \mathbb{R} \backslash\{0\}\right\}=\infty
$$

for each additive mapping $T: G \rightarrow E$.

(ii) If $G=\left(\mathbb{R},\|\cdot\|_{1}\right)$ with the $\beta$-homogeneous norm $\|\cdot\|_{1}=|\cdot|^{\beta}$ and $E=\left(\mathbb{R},\|\cdot\|_{2}\right)$ with the Euclidean metric $\|\cdot\|_{2}=|\cdot|$, then

$$
\|f(x+y)-f(x)-f(y)\|_{2} \leq \varepsilon\left(\|x\|_{1}^{\frac{1}{\beta}}+\|y\|_{1}^{\frac{1}{2 \beta}}\right)
$$

for any $x, y \in \mathbb{R}$, however

$$
\sup \left\{\frac{\|f(x)-T(x)\|_{2}}{\|x\|_{1}^{\frac{1}{\beta}}}: x \in \mathbb{R} \backslash\{0\}\right\}=\infty
$$

for each additive mapping $T: G \rightarrow E$.

(iii) If $G=\left(\mathbb{R},\|\cdot\|_{1}\right)$ with the $\beta_{1}$-homogeneous norm $\|\cdot\|_{1}=|\cdot|_{1}^{\beta}$ and $E=\left(\mathbb{R},\|\cdot\|_{2}\right)$ with the $\beta_{2}$-homogeneous norm $\|\cdot\|_{2}=|\cdot|_{2}^{\beta}$, then

$$
\|f(x+y)-f(x)-f(y)\|_{2} \leq \varepsilon^{\beta_{2}}\left(\|x\|_{1}^{\frac{\beta_{2}}{\beta_{1}}}+\|y\|_{1}^{\frac{\beta_{2}}{2 \beta_{1}}}\right)
$$

for any $x, y \in \mathbb{R}$, however

$$
\sup \left\{\frac{\|f(x)-T(x)\|_{2}}{\|x\|_{1}^{\frac{\beta_{2}}{\beta_{1}}}}: x \in \mathbb{R} \backslash\{0\}\right\}=\infty
$$

for each additive mapping $T: G \rightarrow E$.

Remark 3. Set $\mu=\frac{\varepsilon}{8}$. By using a similar proof as in the Theorem 3.1 for Jensen's equation, we can also get

$$
\left|2 f\left(\frac{x+y}{2}\right)-f(x)-f(y)\right| \leq \varepsilon\left(|x|+|y|^{\frac{1}{2}}\right),
$$

however

$$
\sup \left\{\frac{|f(x)-T(x)|}{|x|}: x \in \mathbb{R} \backslash\{0\}\right\}=\infty
$$

for each additive mapping $T: \mathbb{R} \rightarrow \mathbb{R}$.

Thus, we can obtain a conclusion similar to remark 2 relating to Jensen's equation. This leads to the fact that the stability of Jensen's equation does not hold as long as one of the numbers $p, q$ equals $\beta, \frac{1}{\beta}$ or $\frac{\beta_{2}}{\beta_{1}}\left(0<\beta_{1}, \beta_{2} \leq 1\right)$. 
In summary, under the condition that $G$ and $E$ are $F$-spaces with certain property, one is interested to prove that the Hyers-Ulam-Rassias stability is fulfilled in three cases: $\left(\triangle_{1}\right) p, q<\beta_{2}$ (see [24]), $\left(\triangle_{2}\right) p, q>\frac{1}{\beta_{1}}$ (see [24]) and $\left(\triangle_{3}\right) \beta_{2}<p, q<\frac{1}{\beta_{1}}\left(p, q \neq \frac{\beta_{2}}{\beta_{1}}\right)$, but this fails as long as $p$ or $q$ is equal to $\beta_{2}, \frac{1}{\beta_{1}}$ or $\frac{\beta_{2}}{\beta_{1}}\left(0<\beta_{1}, \beta_{2} \leq 1\right)$.

\section{References}

[1] S. Czerwik, Functional Equations and Inequalities in Several Variables, World Scientific Publishing Co., Inc., River Edge, NJ, 2002.

$[2]$, Stability of Functional Equations of Ulam-Hyers-Rassias Type, Hadronic Press,Inc., Florida, 2003.

[3] Z. Gajda, On stability of additive mappings, Internat. J. Math. Math. Sci. 14 (1991), no. 3, 431-434.

[4] P. Găvrută, A generalization of the Hyers-Ulam-Rassias stability of approximately additive mappings, J. Math. Anal. Appl. 184 (1994), no. 3, 431-436.

[5] R. B. Holmes, Geometric Functional Analysis and Its Applications, Graduate Texts in Mathematics, No. 24. Springer-Verlag, New York-Heidelberg, 1975.

[6] D. H. Hyers, On the stability of the linear functional equation, Proc. Nat. Acad. Sci. U.S.A. 27 (1941), 222-224.

[7] D. H. Hyers, George Isac, and Th. M. Rassias, Stability of Functional Equations in Several Variables, Birkhäuser, Boston, 1998.

[8] S.-M. Jung, Hyers-Ulam-Rassias stability of Jensen's equation and its application, Proc. Amer. Math. Soc. 126 (1998), no. 11, 3137-3143.

[9] Z. Kominek, On a local stability of the Jensen functional equation, Demonstratio Math. 22 (1989), no. 2, 499-507.

[10] Y.-H. Lee and K.-W. Jun, A generalization of the Hyers-Ulam-Rassias stability of Jensen's equation, J. Math. Anal. Appl. 238 (1999), no. 1, 305-315.

[11] J. C. Parnami and H. K. Vasudeva, On Jensen's functional equation, Aequationes Math. 43 (1992), no. 2-3, 211-218.

[12] Th. M. Rassias, New generalizations of Jensen's functional equation, Proc. Amer. Math. Soc. 123 (1995), no. 2, 495-503.

[13] _ On the stability of the linear mapping in Banach spaces, Proc. Amer. Math. Soc. 72 (1978), no. 2, 297-300.

[14] _ On a modified Hyers-Ulam sequence, J. Math. Anal. Appl. 158 (1991), no. 1, $106-113$.

[15] - On the stability of functional equations and a problem of Ulam, Acta Appl. Math. 62 (2000), no. 1, 23-130.

[16] Th. M. Rassias and P. Šemrl, On the Hyers-Ulam stability of linear mappings, J. Math. Anal. Appl. 173 (1993), no. 2, 325-338.

[17] _ On the behavior of mappings which do not satisfy Hyers-Ulam stability, Proc. Amer. Math. Soc. 114 (1992), no. 4, 989-993.

[18] S. Rolewicz, Metric Linear Spaces, PWN-Polish Scientific Publishers, Warsaw, 1972.

[19] T. Trif, Hyers-Ulam-Rassias stability of a Jensen type functional equation, J. Math. Anal. Appl. 250 (2000), no. 2, 579-588.

[20] S. M. Ulam, Problems in Modern Mathematics, Science Editions John Wiley \& Sons, Inc., New York 1964.

[21] J. Wang, The additive approximation on a four-variate Jensen-type operator equation, Int. J. Math. Math. Sci. (2003), no. 50, 3171-3187.

[22] _ Some further generalizations of the Hyers-Ulam-Rassias stability of functional equations, J. Math. Anal. Appl. 263 (2001), no. 2, 406-423. 
[23] - On the generalizations of the Hyers-Ulam-Rassias stability of Cauchy equations, Acta Anal. Funct. Appl. 4 (2002), no. 4, 294-300.

[24] $\quad$ On the generalizations of the stability of Pexider equations and Jensen equations, Nonlinear Funct. Anal. Appl. 7 (2002), no. 2, 229-239.

[25] A. Wilansky, Modern Methods in Topological Vector Spaces, McGraw-Hill, New York, 1978.

DONGYAN ZHANG

Science Institute

INFORMATION ENGINEERING UNIVERSITY

Zhengzhou 450001, China

E-mail address: zdymath@126.com

JIAN WANG

Department of Mathematics

Fujian Normal University

Fuzhou 350007, China 